\title{
Estudo da presença de fatores de riscos intrínsecos para quedas, em idosos institucionalizados
}

\author{
Study of intrinsic risk factors for falls in institutionalized elderly \\ people
}

Ruth Losada de M enezes ${ }^{1}$

$\mathrm{M}$ aria $\mathrm{M}$ árcia Bachion ${ }^{2}$

${ }^{1}$ Departamento de Enfermagem eFisioterapia, Universidade Católica de Goiás. Av. Universitária 1440, Setor Universitário. 74605-010 Goiânia GO. ruthlosada@uol.com.br

${ }^{2}$ Faculdade deEnfermagem, UniversidadeFederal de Goiás.
Abstract The objective of this research was to identify intrinsic risk factors that predispose elderly people living in long-term institutions in the city of Goiânia (GO) to falls. The present descriptive transversal study was carried out in six longterm institutions for seniors in the city of Goiânia. Theinvestigated sample consisted of 95 elderly that fitted the inclusion and exclusion criteria. A questionnaire was used for collecting clinical data related to health-disease conditions; cognitive data; capacity to develop basic daily life activities and equilibrium and gait. In general terms, as pointed out the literature, the examined elderly presented different fall risk factors such as: motor difficulty in lower limbs (90\%), visual deficit $(81,1 \%)$, use of 3 or morekinds of medicines $(59,7 \%)$, suspected depression (37,9\%), lack of equilibrium/unstable unipodal support $(37,9 \%)$ abnormally decreased height in step $(32,6 \%)$. The obtained data allow us to set indicators for the increase in falls among the studied sample, demonstrating the need for creating strategies for health promotion, prevention of injuries and rehabilitation.

Key words Fall accidents, Elderly, Risk factors
Resumo Esta pesquisa teve como objetivo identificar a presença de fatores intrínsecos que predispõem a quedas em idosos moradores em instituiç̃̃es de longa permanência na cidade de Goiânia (GO). Trata-se de estudo descritivo transversal realizado em seis instituições de longa permanência para idosos existentes na cidade de Goiânia. A amostra da investigação constou de 95 idosos que atenderam aos critérios de inclusão e exclusão. U tilizou-se um questionário contendo dados clínicos relacionados às condições de saúde doença; dados cognitivos; avaliação da capacidade para as atividades básicas de vida diária e avaliação do equilíbrio e marcha. Os idosos avaliados, no geral, apresentam vários fatores de risco, apontados pela literatura, para quedas, tais como: relato de dificuldade motora em membros inferiores (90\%), déficit visual $(81,1 \%)$, uso detrês ou mais medicamentos (59,7\%), suspeita de depressão (37,9\%), falta de equilíbrio em apoio unipodal $(37,9 \%)$ e altura do passo anormalmentediminuída (32,6\%) . Asinformações obtidas nos permitem apontar condições determinantes que aumentam a possibilidade dos eventos queda acontecerem na amostra estudada, sinalizando à necessidade de que estratégias de promoção de saúde, prevenção deagravos e reabilitação devam ser tomadas.

Palavras-chave Acidentes por quedas, Idosos, Fatores de risco 
Introdução

Queda pode ser definida como um deslocamento não-intencional do corpo para um nível inferior à posição inicial, com incapacidade de correção em tempo hábil, determinado por circunstâncias multifatoriais que comprometem a estabilidade ${ }^{1}$.

As causas que provocam as quedas são múltiplas e podem ser agrupadas em fatores intrínsecos e extrínsecos. Entre os primeiros, encontram-se as alterações fisiológicas pelas quais o idoso passa, condições patológicas e efeitos adversos de medicações; ou uso concomitante de medicamentos. Entre os fatores extrínsecos, destacam-se os perigos ambientais e calçados inadequados². A maioria das quedas apresentadas pe los idosos resulta de uma interação complexa entre estes fatores, comprometendo os sistemas envolvidos com a manutenção do equilíbrio.

A queda é um evento comum para a maioria das pessoas idosas e pode ter conseqüências desastrosas. No Brasil, segundo dados do Sistema de Informação M édica/M inistério da Saúde, entreos anos de 1979 e 1995, cerca de 54.730 pessoas morreram devido a quedas, sendo que $52 \%$ delas eram idosos, com $39,8 \%$ apresentando idade entre 80 anos e 89 anos $^{3}$. A participação das quedas na mortalidade proporcional por causas externas no Brasil, entre os anos de 1984 a 1994, cresceu de $3 \%$ para $4,5 \%{ }^{4}$.

No Brasil, em um estudo epidemiológico realizado com idosos da comunidade residentes na cidade de São Paulo, a prevalência de quedas foi de $30 \%$ e de quedas recorrentes, cerca de $11 \% 4$. Entre idosos residentes em instituições de longa permanência em outros países, esta prevalência pode subir para cerca de $60 \%$ a $75 \%{ }^{5}$. Estudos acerca da prevalência de quedas em idosos institucionalizados no Brasil ainda são escassos.

A associação significativa entre presença de incapacidade funcional e fragilidade nestes locais fica evidenciada pela alta incidência de quedas. Fatores como idade avançada, presença de doenças crônicas e a imobilidade são fortes indicadores de risco aumentado para quedas ${ }^{6}$. Os principais fatores de risco para quedas em idosos são: idadeavançada, sexo feminino, função neuromuscular prejudicada, presença de doenças crônicas, história prévia de quedas, prejuízos psicocognitivos, polifarmácia, uso de benzodiazepínicos, pre sença de ambientefísico inadequado, incapacidade funcional e hipotensão postural ${ }^{7}$. 0 risco de cair pode crescer com o acúmulo desses fatores, sugerindo que as quedas sejam resultado de um efeito acumulado de múltiplas debilidades ${ }^{8}$.
As conseqüências e os custos envolvidos com as quedas em idosos são relevantes tanto para o indivíduo, em termos dos traumas físicos e psicológicos, da perda de independência e até mesmo do risco de morte, quanto para os serviços de saúde, em termos de utilização de recursos e ocupação de leitos hospitalares 9 . 0 custo dessas quedas torna-se expressivo e maior quando 0 idoso édependente ou passa a necessitar de institucionalização ${ }^{3}$.

A morbidade relacionada às quedas tem várias implicações além das fraturas. Podem causar prejuízos físicos e psicológicos. 0 medo de cair novamente pode ser a complicação mais incapacitante de uma queda, gerando uma diminuição da mobilidade e aumentando o desuso. Um evento de queda pode desencadear uma série de complicações, com conseqüente perda da capacidadefuncional, aumentan do a susceti bilidade a um novo evento no futuro ${ }^{6}$.

Embora seja evidente 0 aumento e importância do evento queda entre a população idosa, encontram-se poucos estudos epidemiológicos sobre esse assunto, especial mente em idosos residentes em instituições de longa permanência.

Esta pesquisa teve como objetivo identificar a presença de fatores intrínsecos que predispõem a quedas em idosos moradores em instituições delonga permanênciana cidadedeGoiânia(GO).

Espera-se que os resultados encontrados nesta pesquisa possam subsidiar o planejamento de medidas preventivas a serem implantadas pelas instituições, visando à melhora da qualidade de vida desta população, bem como gerar dados para estudos futuros, não só na cidade de Goiânia mas em outras localidades.

\section{Casuística emétodos}

Esta pesquisa descritiva transversal foi realizada junto a idosos moradores em instituições delonga permanência na cidade de Goiânia.

Na cidade, no primeiro semestre de 2004 , funcionavam oito instituições de longa permanência para idosos, todas classificadas como filantrópicas (sem fins lucrativos) e com alvará de funcionamento, registradas na 65a Promotoria de Justiça do M inistério Público do Estado de Goiás, órgão que trata de assuntos relacionados aos idosos.

Durante a seleção dos locais de estudo, no início de 2005, uma das instituições foi fechada por não apresentar condições econômicas para o funcionamento eatendimento aos idosos, sen- 
do estes encaminhados para outras instituições e/ou para seus familiares responsáveis.

Uma instituição foi excluída por abrigar moradores com intenso déficit cognitivo, impedindo a decisão livre eesclarecida dos mesmos sobre a sua participação no estudo.

D esta forma, a pesquisa foi realizada em seis instituições de longa permanência para idosos, mediante aprovação dos responsáveis pelas mesmas edo Comitêde Ética em Pesquisa da U niversidadeFederal de Goiás. Ao aceitar participar do estudo, os sujeitos assinaram o termo de consentimento livre e esclarecido, sendo garantido sigilo e anonimato aos participantes.

No início da pesquisa, 250 pessoas moravam nas seis instituições de longa permanência para idosos, que foram cenários do estudo.

Para seleção dos participantes, foram adotados os seguintes critérios de inclusão: ter 60 anos ou mais, morar por no mínimo doze meses na mesma instituição e deambular, mesmo com auxílio de dispositivo de auxílio à marcha.

Foram excluídos os idosos que apresentavam déficits cognitivos e déficit visual ou auditivo severamente limitante e não compensado por uso de óculos ou aparelho de amplificação sonora, dificultando ou impedindo a realização dos testes de avaliação propostos. Dessa forma, foram incluídos 95 sujeitos no estudo.

Como instrumento de coleta de dados, foi utilizado um questionário com dados clínicos relacionados às condições de saúde-doença. As variáveis estudadas envolveram doenças crônicas referidas (reumatismo, asma, hipertensão arterial, insuficiência vascular, diabetes, obesidade, história deacidentevascular encefálico prévio, incontinência urinária, obstipação intestinal, problemas para dormir, catarata, problemas de coluna e asma); uso de dispositivos para auxílio da marcha; as condições percepto-sensoriais, avaliadas através das variáveis de problemas com a visão e audição; número de medicações em uso regular; tipos de medicamentos em uso; realização regular deexercícios físicos; suspeita de depressão; capacidade para as atividades básicas de vida diária e avaliação do equilíbrio e marcha. Para investigação de suspeita de depressão, capacidade para as atividades de vida diária e avaliação de equilíbrio e marcha, foram utilizados os seguintes instrumentos, respectivamente: escala de depressão geriátrica abreviada de Yesavage - GDS ${ }^{10}$, escala de ativida- des básicas de vida diária de Katz ${ }^{11}$ e escala "Performance - Oriented Mobility Assessment" (POMA), versão POMA - Brasil'12.

Os dias das en trevistas foram agendados com os responsáveis pelas instituições, os horários para abordagem dos idosos foram escolhidos de acordo com a rotina de cada local e disponibilidade dos pesquisadores. Os idosos foram avaliados em locais variados das instituições, mais freqüentemente nos quartos e áreas externas.

A coleta de dados foi realizada nos meses de janeiro a junho de 2005 pela pesquisadora e por dois auxiliares de pesquisa (acadêmicos do quinto e oitavo período do curso de graduação em Fisi oterapia da Universidade Católica de Goiás). Os auxiliares de pesquisa foram submetidos a um treinamento, acompanhando uma das pesquisadoras, por um período suficiente para a avaliação dos idosos de uma primeira instituição. Ao longo do processo de avaliação dos onze primeiros idosos integrantes da lista de amostragem selecionada, foram identificadas necessidades de pequenos ajustes no questionário, que, desta forma, foi refinado. Neste período, foram esclarecidas todas as dúvidas dos pesquisadores, e, quando necessário, os idosos foram reavaliados de modo a garantir a uniformização do procedimento de coleta de dados.

O procedimento deavaliação foi realizado da seguinte forma: com o idoso sentado em uma cadeira, foram feitas algumas questões sobre episódios de queda e seus contextos e condições de saúde-doença. A pós a coleta destes dados, um dos pesquisadores aplicou a escala de atividades básicas de vida diária de Katz ${ }^{11}$.

Após, seguiram-seostestesfuncionais deequilíbrio emarcha, sempre com a presença do auxiliar ou funcionário da instituição, para garantir segurança às manobras, seguindo-se o protocolo de recomendações de aplicação ${ }^{12}$.

A última avaliação foi a cognitiva, abordando a suspeita de depressão através da escala de depressão geriátrica - abreviada de Yesavage ${ }^{10}$. As perguntas desta escala muitas vezes sensibilizam ou despertam longas narrativas pelos idosos, por isso mesmo foi selecionada, para meIhor andamento da coleta de dados, para ser 0 último aspecto a ser avaliado.

Para a análise de dados, foram utilizados procedimentos de estatística descritiva, através do Sistema Sphinx versão 4.5. 


\section{Resultados}

Dos 95 idosos, 50 (52,6\%) eram do sexo feminino e a faixa etária mais freqüente foi de 70-79 anos $(47,4 \%)$, seguido de 80 ou mais $(28,4 \%)$ e 60-69 anos (24,2\%).

Os problemas de saúde relatados são apresentados na Figura 1. Destacaram-se "pressão alta" (58,9\%), "problemas de coluna" (50,5\%) e catarata (45,3\%). É oportuno lembrar que cada respondente poderia alegar mais de um problema de saúde e que idosos com déficit visual e/ou auditivo severos não foram incluídos no estudo.

Dos 95 idosos entrevistados, 14 (14,8\%) utilizavam dispositivos para auxílio de marcha, sendo que $13(92,8 \%)$ utilizam bengala e $1(7,1 \%)$ utiliza andador.

Quanto às condições percepto-sensoriais, foi relatado e/ou observado em $77(81,1 \%)$ dos idosos déficit visual. Destes, $45(58,4 \%)$ apresentaram déficit corrigido com órtese e 32 (41,5\%) déficit não corrigido. Quanto à audição, 77 (81\%) dos idosos referiram não apresentar déficit auditivo.

0 uso regular de medicações prescritas por médicos esteve presente em 87 (91,6\%) dos idosos entrevistados. Quanto ao número de medicamentos usados, observou-se que 52 idosos
(59,7\%) faziam uso de três ou maisfármacos, 35 $(40,2 \%)$ faziam uso de 1 -2 fármacos esomente 8 $(8,4 \%)$ não faziam uso de medicamentos.

Os grupos de medicamentos mais utilizados pelos idosos são exibidos na Figura 2, que descreve as classes de medicamentos utilizadas e seu perfil de uso na amostra. A maior parte dos entrevistados consumia drogas para problemas cardiovasculares. Na categoria outros, incluem-se: laxantes, anal gésicos, antidiabéticos e antipsicóticos.

Do total de idosos entrevistados, 59 (62,1\%) não realizam exercíciosfísicos regulares. 0 relato de dificuldade motora em membros inferiores foi manifestado por 86 (90\%) dos idosos participantes.

No presenteestudo, a suspeita de depressão foi encontrada em 36 (37,9\%) idosos entrevistados.

Em relação a atividades básicas de vida diária, 92,6\% dos idosos que participaram da pesquisa apresentaram independência para cinco a seis atividades, evidenciando uma amostra com perfil diferenciado, ou seja, independentefuncionalmente, o que já era esperado, pois para participar do estudo deveriam apresentar deambulação (mesmo que com auxílio à marcha), não apresentar déficit cognitivo elimitações perceptivo-sensoriais (visão e audição).

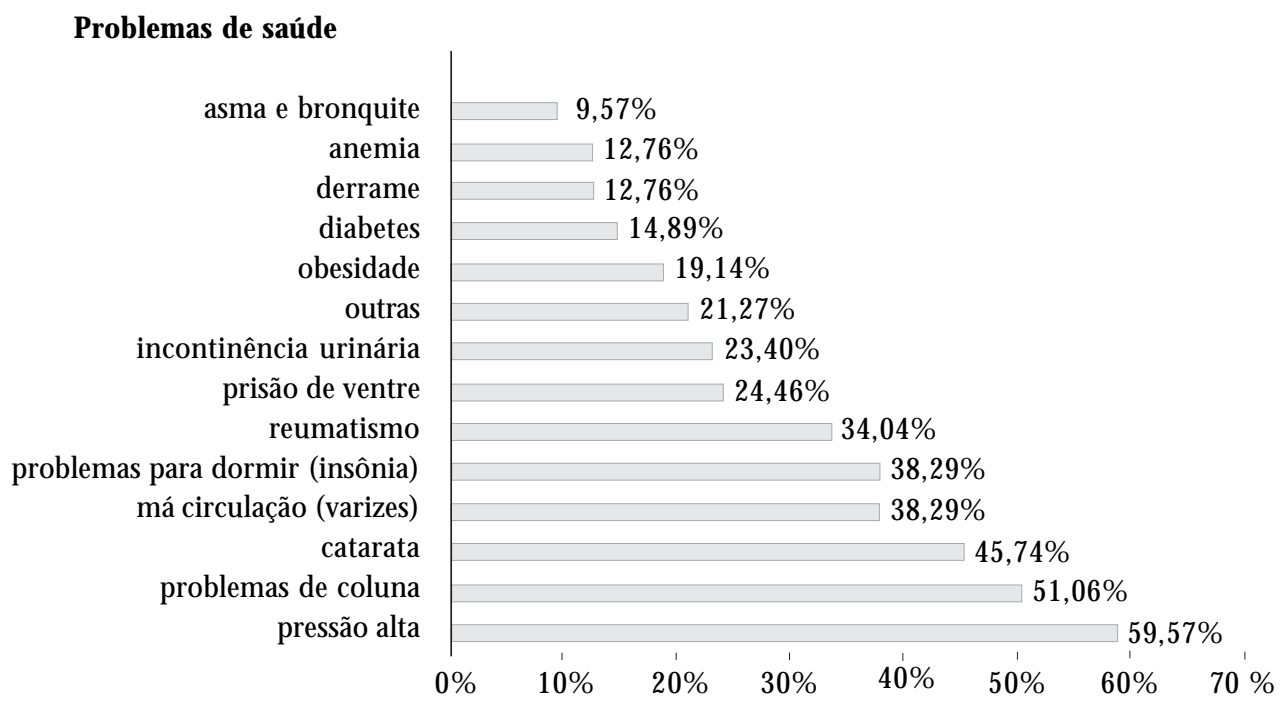

Figura 1. Problemas de saúde relatados pelos idosos institucionalizados. Goiânia (GO), 2005. 
Analisando as manobras de equilíbrio, foi possível verificar algumas alterações que merecem ser destacadas (Tabela 1).

Cumpre ressaltar que "se levantar da cadeira" e "sentar" apresentou desempenho adaptativo para mais da metade dos idosos. 0 "equilíbrio em apoio unipodal" foi adaptativo ou anormal para aproximadamente dois terços dos ido- sos e o "alcançar para cima", adaptativo ou anormal para aproximadamente metade dos idosos.

A Tabela 2 expõe manobras relacionadas à avaliação da marcha, chamando-se a atenção para a manobra "altura do passo", a qual 32,6\% dos idosos desenvolveram de forma anormal, ou seja, mais baixo do que o necessário, chegando a arrastar os pés.

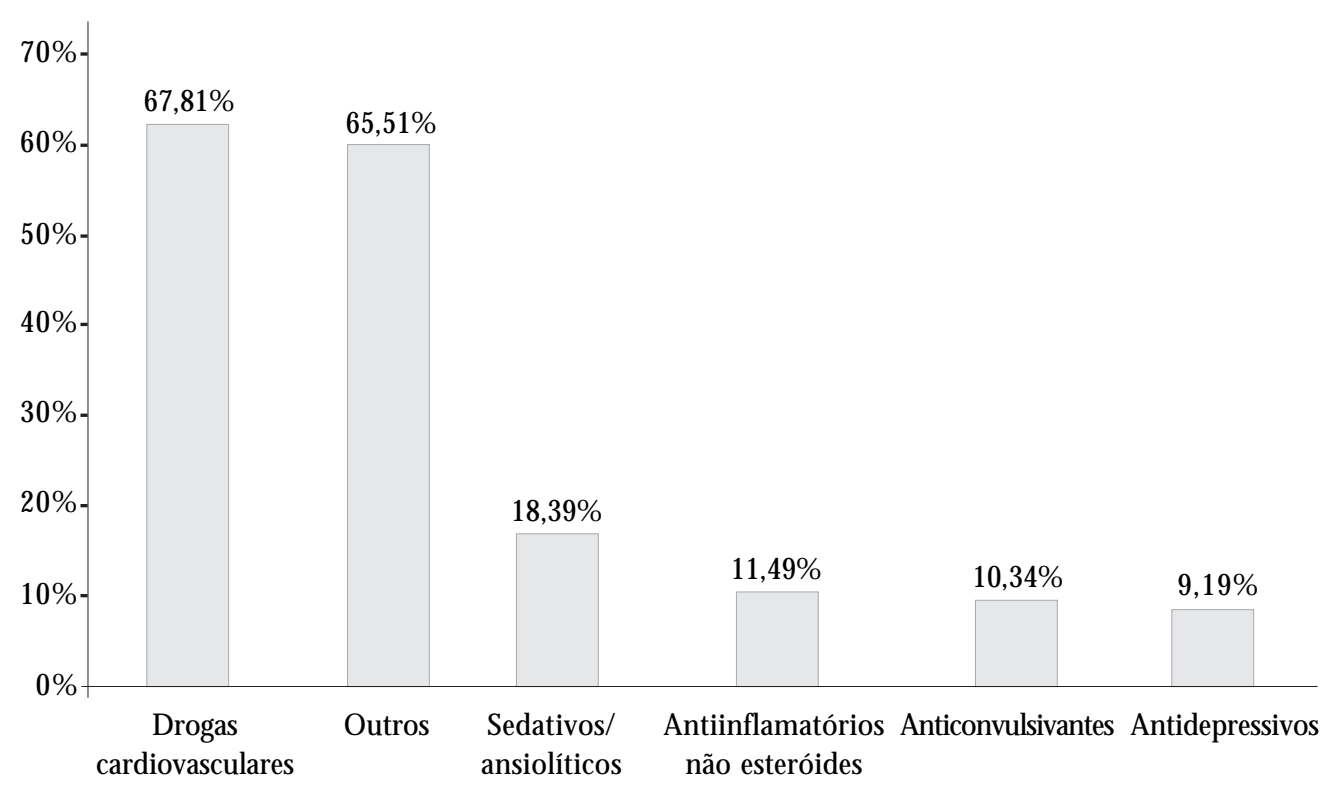

M edicamentos em uso

Figura 2. Grupo de medicamentos em uso pelos idosos institucionalizados. Goiânia (GO), 2005.

Tabela 1. Desempenho dos idosos institucionalizados nas manobras de avaliação do equilíbrio do POM A Brasil. Goiânia (GO), 2005.

\begin{tabular}{|c|c|c|c|c|c|c|c|c|}
\hline \multirow[t]{3}{*}{ Manobras } & \multicolumn{6}{|c|}{ Categorias } & \multicolumn{2}{|c|}{ Total } \\
\hline & \multicolumn{2}{|c|}{ normal } & \multicolumn{2}{|c|}{ adaptativa } & \multicolumn{2}{|c|}{ anormal } & \multirow[b]{2}{*}{$\mathrm{N}^{\circ}$} & \multirow[b]{2}{*}{$\%$} \\
\hline & $\mathrm{N}^{\circ}$ & $\%$ & $\mathrm{~N}^{\circ}$ & $\%$ & $N^{\circ}$ & $\%$ & & \\
\hline Levantando-se da cadeira & 32 & 33,7 & 61 & 64,2 & 2 & 2,1 & 95 & 100 \\
\hline Equilíbrio em apoio unipodal & 26 & 27,4 & 33 & 34,7 & 36 & 37,9 & 95 & 100 \\
\hline Alcançar para cima & 50 & 52,6 & 24 & 25,3 & 21 & 22,1 & 95 & 100 \\
\hline Sentar & 24 & 25,3 & 69 & 72,6 & 2 & 2,1 & 95 & 100 \\
\hline
\end{tabular}


Tabela 2. Desempenho dos idosos institucionalizados nas manobras de avaliação da marcha do POM A Brasil. Goiânia (GO), 2005.

\begin{tabular}{|c|c|c|c|c|c|c|}
\hline \multirow[t]{3}{*}{ Manobras } & \multicolumn{4}{|c|}{ Categorias } & \multicolumn{2}{|c|}{ Total } \\
\hline & \multicolumn{2}{|c|}{ normal } & \multicolumn{2}{|c|}{ anormal } & \multirow[b]{2}{*}{$\mathrm{N}^{\circ}$} & \multirow[b]{2}{*}{$\%$} \\
\hline & $\mathrm{N}^{\circ}$ & $\%$ & $\mathrm{~N}^{\circ}$ & $\%$ & & \\
\hline Altura do passo & 64 & 67,4 & 31 & 32,6 & 95 & 100 \\
\hline Continuidade do passo & 71 & 74,7 & 24 & 25,3 & 95 & 100 \\
\hline Estabilidade de tronco & 72 & 75,8 & 23 & 24,2 & 95 & 100 \\
\hline Sustentação durante a marcha & 68 & 71,6 & 27 & 28,4 & 95 & 100 \\
\hline Virando durante a marcha & 75 & 78,9 & 20 & 21,1 & & \\
\hline
\end{tabular}

\section{Discussão}

A hipertensão arterial sistêmicaéa doença crônica mais comum entre os idosos, sendo que sua prevalência aumenta progressivamente com a idade e consiste no principal fator de risco para os acidentes vasculares cerebrais. Alguns estudos na literatura apontam a história pregressa de acidente vascular cerebral como fator associado a quedas ${ }^{13,14}$. Possivelmente pelas conseqüências desteevento, a hemiplegia ou paresia da extremidade inferior afeta a marcha do indivíduo, que assume uma posição ereta instável com comprometimento da base de apoio e com tendência a cair para frente, do lado enfraquecido. Também, 0 aparecimento de disfunção visual e lesão espacial-visual em seguida ao "derrame" são bastante comuns e podem influenciar o equilíbrio do idoso e a mobilidade segura ${ }^{15}$.

Em outro extremo, temos a hipotensão ortostática também comum nesta faixa etária, tornando-os vulneráveis às quedas. Muitos idosos são capazes de conservar o controle postural, mas se passarem pelo desafio de condições patológicas que interfiram na regulação do controle da pressão sangüínea, a probabilidade de instabilidade postural e quedas é grande ${ }^{15}$.

Distúrbios músculo-esqueléticos, como osteoartrose, que resultam em rigidez e dor nas articulações, estão ligadas à instabilidade no caminhar e no equilíbrio ${ }^{15}$. A perda da força muscular conseqüente a estes distúrbios pode prejudicar a mobilidade, levando à dependência funcional, sendo esta uma condição relacionada com o evento queda.
Para Tinettiti ${ }^{16}$, as doenças crônicas e as deficiênciasfísicas presentesnosidosos contribuem para a ocorrência de quedas. Esta autora também relata que o risco de queda aumenta linearmente com o número de anormalidades existentes.

Pequena porcentagem dos idosos avaliados utilizava dispositivos para auxílio de marcha (14,8\%). Estudos revelaram correlação entre a utilização do dispositivo para auxílio de marcha e a presença de quedas ${ }^{17,18}$.

A tecnologia assistiva possibilita a promoção da independência funcional, facilitando a realização das atividades cotidianas pelosidosos. Esta tecnologia pode ser empregada para dar apoio quando uma pessoa anda ese equilibra, mas pode ter efeito oposto e contribuir para uma mobilidade insegura e risco de quedas quando da sua ausência em casos de indicação e/ou falhas em seu projeto e/ou utilização inadequada.

A população estudada apresentou um significativo número de idosos com déficit visual não corrigido com órtese $32(33,7 \%)$, sendo este fato um alerta para o risco eminente para futuros episódios de queda.

Inúmeras alterações fisiológicas relacionadas à idade podem afetar o desempenho visual elevar ao risco de quedas. 0 déficit na acuidade visual, restrição do campo visual, aumento da suscetibilidade à luz, percepção de profundidade deficiente ou instabilidade na fixação do olhar constituem-se em fatores relacionados à perda de equilíbrio, uma vez que a visão constitui 0 órgão sensorial que fornece importantes informações aferentes para o mecanismo de controle postural. No estudo de Perracini e Ramos ${ }^{19}$, Cu- 
jos objetivos foram identificar os fatores associados a quedas e às quedas recorrentes em idosos vivendo na comunidade, os idosos com leve e severa diminuição na função visual apresentaram, respectivamente, 1,4 e 2,2 vezes mais riscos de quedas.

Os déficits visuais, quando associados a pouca iluminação ambiental, podem predispor a escorregões e derrapadas em ambientes com irregularidades no chão, tais como poças d'água, pontas de tapetes erguidas, entre outros.

No presente estudo, os idosos referem pequeno ou leve déficit auditivo (19\%). A literatura aponta que a diminuição da sensibilidade auditiva resulta em vertigens e dificulta o controle postural, principalmente em movimentos bruscose mudanças de direção, favorecendo, portanto, a ocorrência de quedas².

As mudanças fisiológicas relacionadas ao envelhecimento possuem uma série de alterações que interferem diretamente nos processos de absorção, distribuição, metabolização e eliminação dos medicamentos ${ }^{20}$. Os medicamentos são utilizados para tratar e reduzir a morbidade associada a diversas doenças. Entretanto, o uso concomitante destes medicamentos predispõe 0 idoso aos riscos de seus efeitos aditivos ${ }^{21}$. 0 uso de medicamentos tem sido abordado em vários estudos como um dos fatores de risco para o evento queda. Estudo realizado por Tinetti et al. 22 aponta que as quedas foram mais freqüentes entre os idosos que faziam uso de vários fármacos concomitantemente. Revela-se, então, mais um risco na população estudada, uma vez que o uso regular de medicamentos esteve presente em $87(91,6 \%)$ dos idosos entrevistados, sendo que $52(54,7 \%)$ faziam uso de três ou mais fármacos.

As quedas podem ser induzidas por meio de inúmeros mecanismos e atos diretos e indiretos da medicação utilizada. Drogas como sedativos/ ansiolíticos, antidepressivos e agentes cardiovasculares (tais como diuréticos, anti-hipertensivos, cardiotônicos) podem levar diretamente a quedas, por causarem hipotensão postural, sedação excessiva e diminuição no tempo de reação, dificuldades no equilíbrio eno caminhar, arritmiase danos a um estado de alerta cognitivo ${ }^{15}$.

Os medicamentos cardiovasculares são considerados na predisposição de quedas em idoSOS, em virtude dos efeitos colaterais como bradicardia, hipotensão, sonolência e fadiga ${ }^{23}$. Os resultados obtidos tendem a alertar, de certa forma, para o risco de quedas, uma vez que mais da metade dos entrevistados fazia uso de drogas para problemas cardiovasculares.
0 envelhecimento é caracterizado por um declínio na performance motora e pela diminuição gradual do movimento, sendo a fraqueza muscular um grande contribuinte para o declínio da funcionalidade do idoso ${ }^{24}$.

Uma diminuição da força muscular afeta em última instância a capacidade laboral, a atividade motora e a adaptabilidade ao ambiente, contribuindo para que ocorram instabilidades e quedas em indivíduos idosos. Vários estudos indicam uma correlação entre a fraqueza muscular e as quedas em idosos, sendo que Paschoa ${ }^{25}$ aponta a fraqueza muscular como segunda maior causa de queda, juntamente com os distúrbios do equilíbrio e da marcha. N este aspecto, deve-se então ser chamada a atenção para a população estudada, uma vez que significativo número de idosos não realizam exercíciosfísicos regulares e relatam fraqueza muscular em membros inferiores.

Os idosos institucionalizados, em geral, dispõem de poucas atividades físicas para participarem, o que pode agravar o ciclo: envel hecimento, menor capacidade funcional e sedentarismo.

Brouwer et al. ${ }^{26}$ realizaram um estudo com 38 idosos para determinar a relação dos efeitos de educação e de programa de atividade física na redução de quedas. Estes autores verificaram que as quedas foram significativamente reduzidas naqueles que receberam ambas as intervenções.

É consenso na literatura os ben efíciosfisiológicos advindos com a prática de atividade física, incluindo ganhos de força muscular, melhora do equilíbrio e do desempenho da marcha, aumento da flexibilidade e do $\mathrm{VO}_{2} \max$ (consumo máximo de oxigênio), proporcionando, assim, uma maior independência aos idosos para realizar suas atividades de vida diária. Também é de consenso os seus benefícios psicológicos, tais como meIhora da auto-estima e da autoconfiança, repercutindo numa melhora da qualidade de vida ${ }^{27}$.

A escolha da modalidade deveser individualizada econsiderando as condições e preferências pessoais. Prazer e socialização são reconhecidos como componentes-chave de programas de exercícios físicos; desta forma, atividades alternativas, quando possível, também são recomendadas, como biodança, práticas psicomotoras, yoga, tai-chi-chuan, musicoterapia e praxiterapia (trabalhos manuais), todas integrantes do rol de técnicas de autopercepção de equilíbrio mental e corporal.

Faz-senecessário criar nas instituições estraté gias para aumentar o nível de atividade física dos idosos. As atividades terapêuticas específicas para a terceira idade levam ao resgate e val orização do 
idoso, melhora de sua auto-estima e manutenção de sua participação produtiva na sociedade.

Deacordo com Gazalleet al. ${ }^{28}$, entre os diversos transtornos cognitivos que afetam idosos, a depressão merecea atenção especial, uma vez que apresenta freqüência elevada e conseqüências negativas na vida dos indivíduos acometidos. Trata-se de um fator predisponente à queda bastante conhecido ${ }^{29}$, indicando, assim, uma condição de risco para 37,9\% dos idosos avaliados. Rubenstein e Josephson ${ }^{17}$ identificaram em seu estudo que idosos com depressão têm 2,2 vezes mais probabilidade de caírem se comparados a idosos que não têm depressão.

A velhice para muitas pessoas é percebida como uma fase dolorosa da vida, sem perspectivas e marcada pela presença de doenças e perdas. Através deobservação não sistematizada, foi possível verificar a existência de problemas de comunicação entre os idosos pesquisados. Percebemos que muitos deles vivem juntos, comem juntos, mas não se comunicam. A dificuldade do convívio social dosidosos, quer na família ou na instituição asilar, pode ser conseqüência das alterações fisiológicas efisiopatológicas decorrentes do envel hecimento. Acreditamos que as pessoas envolvidas com os idosos, sejam cuidadores, enfermeiros, médicos, fisioterapeutas ou outros profissionais, devem motivar os mesmos mediante atividades sócio-interativas, no sentido de que estes possam se engajar ativamente, diminuindo os fatores que levam às alterações do processo de envelhecimento, como a inatividade e depressão.

As alterações das manobras de equilíbrio e marcha percebidas prevêem limitações funcionais para estes idosos, como sentar elevantar do vaso sanitário, tomar banho, sair da cama, alcançar objetos em prateleiras altas, subir edescer escadas e outros.

A incapacidade físico-funcional expressa em termos de comprometimento na realização de algumas atividades de vida diária também foi ressaltada em alguns estudos. Langlois et al. ${ }^{30}$ identificaram que, apesar de mais jovens, idosos entre 65 e 79 anos, quando apresentavam comprometimento funcional, tinham uma chance maior de sofrer queda quando comparados a idosos da mesma idade. A identificação precoce do declínio funcional éessencial quando o objetivo é prevenir episódios de queda.

\section{Conclusões}

Ao analisar al gumas condições de saúde doença dos idosos participantes, verificamos um cenário onde coexistem fatores múltiplos de risco intrínsecos para quedas futuras neste grupo: presença de doenças, relato de dificuldade motora em membros inferiores (90\%), uso de dispositivos auxiliares para marcha $(14,8 \%)$, déficit visual $(81,1 \%)$, uso de três ou mais medicamentos (59,7\%), suspeita de depressão $(37,9 \%)$, falta de equilíbrio em apoio unipodal (37,9\%), dificuldade para alcançar para cima $(22,1 \%)$, altura do passo anormalmente diminuída ( $32,6 \%)$, continuidade anormal da passada (25,3\%), falta de equilíbrio do tronco (24,2\%).

As condições de saúde dos idosos revelam a necessidade de avaliação/reavaliação por parte de profissionais da área de geriatria e gerontologia, no âmbito de promoção de saúde, prevenção de agravos e reabilitação. As informações obtidas nos permitem apontar marcadores que aumentam a possibilidade dos eventos queda acontecerem na amostra estudada.

Os efeitos da diminuição natural do desempenho dos idosos e o risco de quedas podem ser atenuados se forem desenvolvidos com os mesmos programas de atenção à saúde que visem à melhoria das capacidades motoras, alcançando de igual forma melhora dos aspectos psicossociais. Estas medidas necessitam de uma abordagem multidimensional, o que somente é possível através da ação integrada e especial izada de uma equipeinterdisciplinar. 


\section{Colaboradores}

$R L$ de M enezes foi responsável pela elaboração, redação e revisão do artigo eM M Bachion participou da orientação da pesquisa e revisão do artigo.

\section{Referências}

1. Studensk S, Wolter L. Instabilidade e quedas. In: Duthie, EH, Katz PR, organizadores. Geriatria prática. 3a ed. Rio de Janeiro: Revinter; 2002. p. 193-200.

2. Rubenstein CM P, Powers CM, Maclean CH. Quality Indicators for the $M$ anagement and Prevention of Falls and Mobility Problems in Vulnerable Elders. Ann Intern Med 2001; 135:686-693.

3. Fabrício SCC, Rodrigues RAP, Costa Júnior ML. Causas e conseqüências de quedas de idosos atendidos em hospital público. Rev. Saúde Pública 2004; 38(1):93-99.

4. Perracini MR. Fatores associados a quedas em uma coorte de idosos residentes do município de São Paulo [tese]. São Paulo (SP): Universidade Federal de São Paulo; 2000.

5. Hoffman MT, Bankes PF, Javed A, Selhat M. Decreasing the incidence of falls in the nursing home in a cost-conscious environment: a pilot study. JAM DA 2003; 4:95-97.

6. Nevitt MC. Falls in the elderly: risk factors and prevention. In: Masdeu JC, Sudarsky L, Wolfson L, editors. Gait disorders of aging: falls and therapeutic strategies. Philadelphia: Lippincott-Raven; 1997. p.13-36.

7. Kron M, Loy S, Sturm E, Nikolaus T, Becker C. Risk indicators for fall in institutionalized frail elderly. Am J Epidemiol 2003; 158:645-653.

8. Nevitt MC, Cummings SR, Kidd S, Black D. Risk factors for recurrent nonsyncopal falls. A prospective study. JAM A 1989; 261(18):2663-2668.

9. Close J, Ellis M, Hooper R, Glucksman E, Jackson $\mathrm{S}$, Swift C. Prevention of falls in the elderly trial (PROFET): a randomised controlled trial. Lancet 1999; 353(9147):93-97.

10. Yesavage JA, Brink TL, Rose TL, Lum O, Huang V, Adey $M$, Leirer VO. Development and validation of a geriatric depression screening scale: a preliminary report. J Psychiatr Res 1982-83; 17(1):37-49.

11. Freitas EV, Miranda RD, Nery M. Parâmetros clínicos do envelhecimento e avaliação geriátrica global. In: Freitas EV, Py L, Cançado FAX, Doll J, Gorzoni ML, organizadores. Tratado de geriatria e gerontologia. Rio de Janeiro: Guanabara-Koogan; 2002. p. 609-617.

12. Gomes GC. Tradução, adaptação transcultural e exame das propriedades de medida da escala "performance-oriented mobility assessment" (POMA) para uma amostragem de idosos brasileiros institucionalizados [dissertação]. Campinas (SP): Universidade Estadual de Campinas; 2003.

13. Campbell AJ, Reinken J, Allan BC, Martinez GS. Falls in old age: a study of frequency and related clinical factors. Age Ageing 1981; 10(4):264-270.

14. Graafmans WC, Ooms ME, H ofstee HM, Bezemer PD, Bouter LM, Lips P. Falls in the elderly: a prospective study of risk factors and risk profiles. Am J Epidemiol 1996; 143(11):1129-1136.

15. Tideiksaar R. As quedas na velhice: prevenção e cuidados. 2a ed. São Paulo: Organização Andrei Editora Ltda; 2003.

16. Tinetti ME. Performance-oriented assessment of mobility problems in elderly patients. J Am Geriatr Soc 1986; 34(2):119-126. 
17. Rubenstein LZ, Josephson KR. The epidemiology of falls and syncope. Clin Geriatr M ed 2002; 18(2):141158.

18. Piton DA. Análise dos fatores de risco de quedas em idosos: estudo exploratório em instituição de longa permanência no município de Campinas [dissertação]. Campinas (SP): Universidade Estadual de Campinas; 2004.

19. Perracini MP, Ramos LR. Fatores associados a quedas em uma coorte de idosos residentes na comunidade. Rev. Saúde Pública 2002; 36(6):709-716.

20. Pereira LRL, Vecchi LUP, Baptista MEC, Carvalho D. Avaliação da utilização de medicamentos em pacientes idosos por meio de conceitos de farmacoepidemiologia e farmacovigilância. Cienc Saude Colet 2004; 9(2):479-481.

21. Nóbrega OT, Karnikowski M GO. A terapia medicamentosa no idoso: cuidados na medicação. Cienc Saude Colet 2005; 10(2):309-313.

22. Tinetti ME, Baker DI, MacAvay G, Claus E, Garrett P, Gottschalk M, Koch M L, Trainor K, Horwitz RI. A multifactorial intervention to reduce the risk of falling among elderly people living in the community. N Engl J Med 1994; 331:821-827.

23. Coutinho ESF, Silva SD. Uso de medicamentos como fator de risco para fratura grave decorrente de queda em idosos. Cad Saúde Pública 2002; 18(5):1359-1366.

24. Wolfson L, Judge J, Whipple R, King M. Strength is a major factor in balance, gait, and the occurrence of falls. J Gerontol A Biol Sci Med Sci 1995; 50(Spec):64-67.

25. Paschoal S. Como reduzir quedas no idoso. In: Jacob Filho W, organizador. Promoção da saúde do idoso. São Paulo: Lemos Editorial/FM USP; 1998. p. 65-75.

26. Brouwer BJ, Walker C, Rydahl SJ, Culham EG. Reducing fear of falling in seniors through education and activity programs: a randomized trial. J Am Geriatr Soc 2003; 51(6):829-834.

27. Freitas EV, Ghorayeb N, Pereira JBM, Ghorayeb C. Atividade física no idoso. In: Freitas EV, Py L, Cançado FAX, Doll J, Gorzoni ML, organizadores. Tratado de geriatria e gerontologia. Rio de Janeiro: Guanabara-Koogan; 2002. p. 857-865.

28. Gazalle FK, Lima MS, Tavares BF, Hallal PC. Sintomas depressivos e fatores associados em população idosa no Sul do Brasil. Rev. Saúde Pública 2004; 38:365-371.

29. Moreland J, Richardson J, Chan DH, O'N eill J, Bellissimo A, Grum RM, Shanks L. Evidence-based guidelines for the secondary prevention of falls in older adults. Gerontology 2003; 49(2):93-116.

30. Langlois JA, Smith GS, Nelson DE, Sattin RW, Stevens JA, De Vito CA. Dependence in activities of daily living as a risk factor for fall injury events among older people living in the community. JAGS 1995; 43(3):275-278.

Artigo apresentado em 18/12/2008

Aprovado em 25/07/2007

Versão final apresentada em 24/08/2007 